УДК 541.123.3

\title{
РАСТВОРИМОСТЬ ФОСФОРА В МОНОАРСЕНИДЕ ОЛОВА
}

\author{
(ㄷ) 2018 Г. В. Семенова, Т. П. Сушкова, Е. Н. Зинченко, С. В. Якунин \\ Воронежский государственный университет, Университетская пл. 1, 394018 Воронеж, Россия \\ e-mail: semen157@chem.vsu.ru
}

Поступила в редакцию 28.08.2018

\begin{abstract}
Аннотация. Для выяснения конфигурации области гомогенности твердого раствора фосфора в моноарсениде олова методом рентгенофазового анализа были исследованы отожженные при температуре 753 К сплавы, принадлежащие политермическим сечениям $\mathrm{SnAs}-\mathrm{Sn}_{4} \mathrm{P}_{3}$, $\mathrm{SnAs}-\mathrm{Sn}_{0.43} \mathrm{P}_{0.57}, \mathrm{SnAs}-\mathrm{SnP}_{3}$, SnAs-P тройной системы $\mathrm{Sn}-\mathrm{As}-\mathrm{P}$. Установлено, что область существования твердых растворов на основе SnAs имеет асимметричную форму, смещенную в сторону избытка летучих компонентов, причем максимальная растворимость фосфора (3040 мол. \%) отвечает направлению политермического сечения $\mathrm{SnAs}-\mathrm{SnP}_{3}$. Сам моноарсенид олова обладает заметной областью гомогенности, о чем свидетельствует разница параметров решетки образцов, чей состав был приближен к противоположным границам области гомогенного существования фазы путем отжига в контакте через паровую фазу с гетерогенными смесями составов $\mathrm{Sn}_{0.39} \mathrm{As}_{0.61}$ и $\mathrm{Sn}_{0.535} \mathrm{As}_{0.465}$.
\end{abstract}

Ключевые слова: фазовые равновесия, твердые растворы, система Sn-As-P.

DOI: https://doi.org/10.17308/kcmf.2018.20/639

\section{ВВЕДЕНИЕ}

В последние годы большое внимание уделяется изучению соединений $\mathrm{A}^{\mathrm{IV}} \mathrm{B}^{\mathrm{V}}$ и твердых растворов на их основе, являющихся перспективными материалами для изготовления молекулярных контейнеров, электродов литий - ионных аккумуляторов и других устройств для преобразования и хранения энергии [1-3]. В тройных системах с анионным замещением, в частности $\mathrm{Ge}-\mathrm{As}-\mathrm{P}, \mathrm{Si}-\mathrm{As}-\mathrm{P}$, существуют широкие области твердофазной растворимости [4]. В системе Sn-As-P установлено наличие непрерывного ряда твердых растворов $\mathrm{Sn}_{4} \mathrm{As}_{3}-\mathrm{Sn}_{4} \mathrm{P}_{3}(\alpha$-твердый раствор) [5], а также существование твердых растворов на основе моноарсенида олова ( $\beta$-твердые растворы) и фосфида $\mathrm{SnP}_{3}$ ( $\gamma$-твердые растворы) [6]. Растворимость фосфора в моноарсениде олова будет зависеть от того, в виде индивидуального простого вещества или в виде какого-либо из фосфидов олова вводится легирующий компонент.

Цель настоящей работы заключалась в исследовании конфигурации области существования твердых растворов фосфора в моноарсениде олова.

\section{ЭКСПЕРИМЕНТАЛЬНАЯ ЧАСТЬ}

Синтез сплавов осуществляли однотемпературным методом, используя фосфор ОСЧ-9-5, олово марки ОВЧ-000 и мышьяк ОСЧ-9-5, очищенный от оксидов сублимацией в вакууме. Для гомогенизации образцов и приведения системы в состояние, близкое к равновесному, образцы отжигали при температуре 753 К в течение 120 ч.

Рентгенофазовый анализ (РФА) проводили на дифрактометре ARL X'TRA в геометрии $\Theta-\Theta$ с фокусировкой по Бреггу-Брентано; использовалась рентгеновская трубка с медным анодом, $\lambda\left(\mathrm{Cu} K_{\alpha 1}\right)=0.1541 \mathrm{Hм} ; \lambda\left(\mathrm{Cu} K_{\alpha 2}\right)=0.1544 \mathrm{нм}$; шаг съемки $0.04^{\circ}$, время выдержки -3 секунды. Погрешность определения межплоскостных расстояний $d_{h k l}$ не превышала $5 \cdot 10^{-4} \mathrm{Hм}$.

\section{РЕЗУЛЬТАТЫ И ИХ ОБСУЖДЕНИЕ}

В системе $\mathrm{Sn}$-As существуют две промежуточные фазы: моноарсенид олова, плавящийся конгруэнтно при температуре $868 \mathrm{~K}$, и арсенид состава $\mathrm{Sn}_{4} \mathrm{As}_{3}$, разлагающийся перитектически при температуре 860.8 К [7]. В работе [8] была определена область гомогенности фазы $\mathrm{Sn}_{4} \mathrm{As}_{3}$ шириной 3 мол. \% (отклонение от стехиометрии в сторону избытка олова), данные о растворимости компонентов в SnAs в литературе отсутствуют. Для оценки того, насколько изменение состава внутри области гомогенности SnAs влияет на величину параметра решетки, был проведен следующий эксперимент. 
Полученный однотемпературным синтезом образец SnAs был разделен на две части. Одну часть помещали в ампулу с эвтектической смесью $(\mathrm{SnAs}+\mathrm{As})$ состава $\mathrm{Sn}_{0.39} \mathrm{As}_{0.61}$ таким образом, чтобы исключить непосредственный контакт твердых фаз. Другую часть образца помещали аналогичным образом в ампулу с гетерофазной смесью ( $\mathrm{SnAs}+$ $\mathrm{Sn}_{4} \mathrm{As}_{3}$ ) состава $\mathrm{Sn}_{0.535} \mathrm{As}_{0.465}$. Обе ампулы вакуумировали до остаточного давления $5 \cdot 10^{-4}$ ГПа и выдерживали при температуре $823 \mathrm{~K}$ в изотермическом участке печи в течение 100 часов для установления равновесия через паровую фазу, после чего проводили рентгенофазовый анализ образцов моноарсенида олова.

По данным РФА, представленным в табл. 1, межплоскостные расстояния, а также рассчитанные параметры кубической решетки образцов SnAs, coстав которых путем отжига с гетерофазными смесями был приближен к противоположным границам области гомогенности, заметно отличаются.

При установлении равновесия моноарсенида олова с гетерофазной смесью ( $\mathrm{SnAs}+\mathrm{As})$ состав фазы SnAs должен соответствовать границе области гомогенности со стороны избыточного содержания мышьяка. Избыток мышьяка приводит к отклонению от стехиометрии, причем можно рассмотреть две схемы его вхождения в решетку кристалла:

$$
\begin{aligned}
1 / 4 \mathrm{As}_{4}(\mathrm{~g}) \leftrightarrow \mathrm{As}_{\mathrm{As}}^{\times}+\mathrm{V}_{\mathrm{Sn}}^{\times} \text {или } & 1 / 4 \mathrm{As}_{4}(\mathrm{~g}) \leftrightarrow \mathrm{As}_{i}^{\times} \\
(\rightarrow \mathrm{SnAs}) & (\rightarrow \mathrm{SnAs})
\end{aligned}
$$

Моноарсенид олова характеризуется гранецентрированной кубической решеткой, при этом на элементарную ячейку приходится 4 атома, а коэффициент компактности составляет $74 \%$, т. е. решетка является плотноупакованной. В такой структуре два типа междоузлий - октаэдрические и менее просторные тетраэдрические. Размер октаэдрического междоузлия можно оценить как радиус сферы, вписанной в октаэдр $r_{\text {окт }}=b \sqrt{6} 6=0.41 b$, где $b$ - ребро октаэдра. Значение $b$ связано с величиной ребра куба как $b=\left(r_{\mathrm{Sn}}+r_{\mathrm{As}}\right) / \sqrt{2}$. Для радиусов олова $r_{\mathrm{Sn}}=0.141$ нм и $r_{\mathrm{As}}=0.125$ нм [9] можно получить значение $r_{\text {окт }}=0.077$ нм. Это значительно меньшая величина по сравнению с радиусом мышьяка, поэтому образование твердого раствора внедрения маловероятно. В этом случае следует предположить, что основными дефектами будут вакансии олова.

Отжиг образца $\mathrm{SnAs}$ в контакте через паровую фазу с гетерофазной смесью $\left(\mathrm{SnAs}+\mathrm{Sn}_{4} \mathrm{As}_{3}\right)$, напротив, должен приводить к обеднению сплава летучим компонентом:

$$
\mathrm{Sn}_{\mathrm{Sn}}^{\times}+\mathrm{As}_{\mathrm{As}}^{\times} \leftrightarrow 1 / 4 \mathrm{As}_{4}(\mathrm{~g})+\mathrm{V}_{\mathrm{As}}^{\times}
$$

или

$$
\mathrm{Sn}_{\mathrm{Sn}}^{\times}+\mathrm{As}_{\mathrm{As}}^{\times} \leftrightarrow 1 / 4 \mathrm{As}_{4}(\mathrm{~g})+\mathrm{Sn}_{i}^{\times}
$$

Учитывая представленные выше значения радиуса олова и размера междоузлия, очевидно, что в этом случае также следует ожидать формирования твердого раствора вычитания.

Известно, что вакансии в кристалле создают поля растяжения, причем в случае больших по размерам вакансий олова это должно приводить к увеличению межатомных расстояний. В случае вакан-

Таблица 1. Межплоскостные расстояния $(d, \AA)$ и параметр решетки $(a, \AA)$ образцов моноарсенида олова,

\begin{tabular}{|c|c|c|c|}
\hline \multicolumn{2}{|c|}{ SnAs (ICDD PDF2) } & \multicolumn{2}{|c|}{$d_{\mathrm{SnAs}}$} \\
\hline hkl & $d_{\mathrm{SnAs}}$ & $\begin{array}{c}\text { после отжига со смесью }(\mathrm{SnAs}+\mathrm{As}) \\
\text { [after annealing with a mixture } \\
\text { (SnAs + As) }]\end{array}$ & $\begin{array}{c}\text { после отжига со смесью } \\
\left(\mathrm{SnAs}+\mathrm{Sn}_{4} \mathrm{As}_{3}\right) \\
{[\text { after annealing with a mixture }} \\
\left.\left(\mathrm{SnAs}+\mathrm{Sn}_{4} \mathrm{As}_{3}\right)\right]\end{array}$ \\
\hline 111 & 3.3060 & 3.30818 & 3.30627 \\
\hline 200 & 2.8620 & 2.86348 & 2.86243 \\
\hline 220 & 2.0240 & 2.02399 & 2.02362 \\
\hline 311 & 1.7260 & 1.72706 & 1.72587 \\
\hline 222 & 1.6520 & 1.65261 & 1.65218 \\
\hline 400 & 1.4310 & 1.43116 & 1.43112 \\
\hline 331 & 1.3129 & 1.31304 & 1.31430 \\
\hline 420 & 1.2800 & 1.27974 & 1.27977 \\
\hline $\begin{array}{l}\text { Параметр решетки, } a \\
{[\text { Lattice parameter, } a]}\end{array}$ & 5.72415 & $5.72570+/-0.0003$ & $5.72489+/-0.0002$ \\
\hline
\end{tabular}
отожженных в контакте через паровую фазу со сплавами $\mathrm{Sn}_{0.535} \mathrm{As}_{0.465}$ и $\mathrm{Sn}_{0.39} \mathrm{As}_{0.61}$

[Table 1. The interplanar spacings $(d, \AA)$ and the lattice parameter $(a, \AA)$ of tin monoarsenide samples annealed in contact through the vapor phase with $\mathrm{Sn}_{0.535} \mathrm{As}_{0.465}$ and $\mathrm{Sn}_{0.39} \mathrm{As}_{0.61}$ alloys] 
сий мышьяка с меньшим радиусом изменение параметра решетки будет менее значительным. Можно отметить, что значения межатомного расстояния и параметра решетки образца с меньшим содержанием мышьяка близки к характеристикам, приводимым для моноарсенида олова в [10], что неудивительно, поскольку получение образца с максимальным содержанием мышьяка требует контроля давления пара. Сплавление стехиометрических количеств компонентов без поддержания определенного давления пара мышьяка всегда будет приводить к обеднению мышьяком и смещению состава в сторону большего содержания олова.

Таким образом, моноарсенид олова, скорее всего, обладает достаточно протяженной областью гомогенности, однако, чтобы «привязаться» к концентрационной шкале необходимы дополнительные исследования.

B тройной системе $\mathrm{Sn}-\mathrm{As}-\mathrm{P}$ за счет растворения фосфора в моноарсениде олова формируется трехкомпонентный твердый раствор $(\beta)$. Конфигурация и протяженность области гомогенности этой фазы должны изменяться в зависимости от направления разреза, по которому идет легирование. Для выяснения максимальной растворимости фосфора в моноарсениде олова были исследованы сплавы, принадлежащие политермическим разрезам SnAs$\mathrm{Sn}_{4} \mathrm{P}_{3}, \mathrm{SnAs}-\mathrm{Sn}_{0.43} \mathrm{P}_{0.57}, \mathrm{SnAs}-\mathrm{SnP}_{3}, \mathrm{SnAs}-\mathrm{P}$.

При исследовании разреза $\mathrm{SnAs}-\mathrm{Sn}_{4} \mathrm{P}_{3}$ рефлексы $\beta$-твердого раствора были обнаружены на дифрактограммах сплавов, содержащих до 60 мол. \% $\mathrm{Sn}_{4} \mathrm{P}_{3}$. С учетом формирования непрерывного твердого раствора $\left(\mathrm{Sn}_{4} \mathrm{As}_{3}\right)_{x}\left(\mathrm{Sn}_{4} \mathrm{P}_{3}\right)_{1-x}$ легирование моноарсенида олова вдоль разреза $\mathrm{SnAs}-\mathrm{Sn}_{4} \mathrm{P}_{3}$ должно сопровождаться формированием вакансий мышьяка, и состав моноарсенида олова должен смещаться к границе области гомогенности со стороны олова:

$$
\begin{aligned}
\mathrm{Sn}_{4} \mathrm{~B}_{3} \leftrightarrow & 4 \mathrm{Sn}_{\mathrm{Sn}}^{\times}+3 \mathrm{~B}_{\mathrm{As}}^{\times}+\mathrm{V}_{\mathrm{As}}^{\times} \\
& (\rightarrow 4 \mathrm{SnAs}),
\end{aligned}
$$

где В - Р или As.

На рис. $1 a$ представлена дифрактограмма образца, отвечающего этому разрезу, с содержанием моноарсенида олова 95 мол. \%. Помимо рефлексов SnAs, смещенных в сторону больших углов, регистрируются пики $\alpha$-твердого раствора, то есть сплав является гетерофазным. Таким образом, растворимость фосфора в моноарсениде олова по направлению разреза $\mathrm{SnAs}-\mathrm{Sn}_{4} \mathrm{P}_{3}$ составляет менее 5 мол. \%.

При вхождении фосфора в решетку SnAs в направлении разреза $\mathrm{SnAs}-\mathrm{Sn}_{0.43} \mathrm{P}_{0.57}$ (в сплаве $\mathrm{Sn}_{0.43} \mathrm{P}_{0.57}$ соотношение количеств олова и фосфора

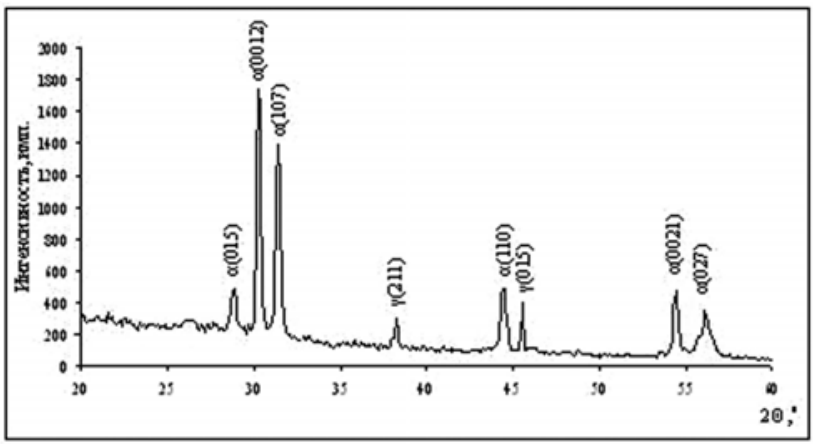

$a$

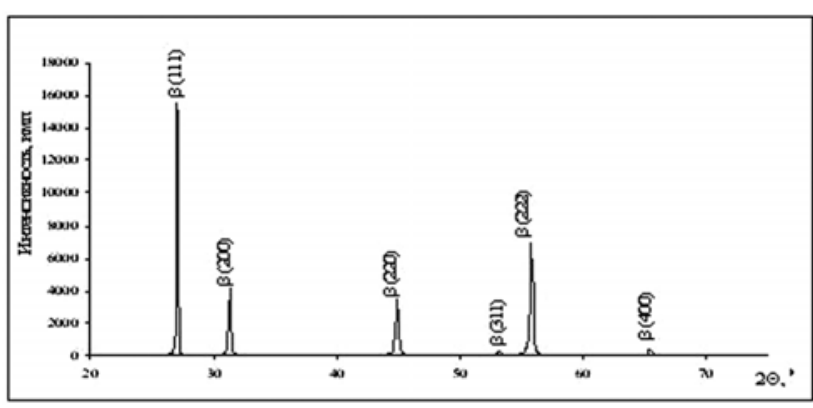

$b$



c

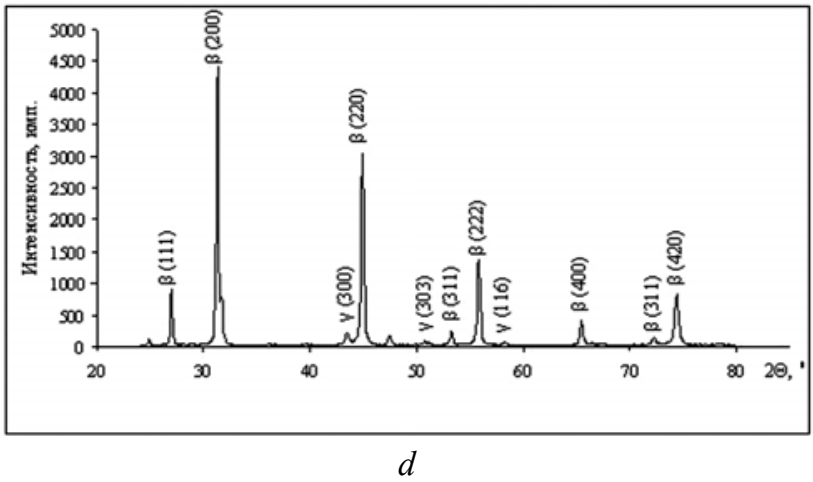

Рис. 1. Дифрактограммы сплавов: $a-(\mathrm{SnAs})_{0.95}\left(\mathrm{Sn}_{4} \mathrm{P}_{3}\right)_{0.05} ; b-(\mathrm{SnAs})_{0.9}\left(\mathrm{Sn}_{0.43} \mathrm{P}_{0.57}\right)_{0.1} ;$ $c-(\mathrm{SnAs})_{0.7}\left(\mathrm{SnP}_{3}\right)_{0.3} ; d-(\mathrm{SnAs})_{0.9} \mathrm{P}_{0.1}$

[Fig. 1. X-ray powder diffraction patterns of alloys: $a-(\mathrm{SnAs})_{0.95}\left(\mathrm{Sn}_{4} \mathrm{P}_{3}\right)_{0.05} ; b-(\mathrm{SnAs})_{0.9}\left(\mathrm{Sn}_{0.43} \mathrm{P}_{0.57}\right)_{0.1}$; $c-(\mathrm{SnAs})_{0.7}\left(\mathrm{SnP}_{3}\right)_{0.3} ; d-(\mathrm{SnAs})_{0.9} \mathrm{P}_{0.1}$ 
составляет 3:4) должно наблюдаться смещение области гомогенности в сторону недостатка олова:

$$
\begin{aligned}
& \mathrm{Sn}_{3} \mathrm{P}_{4} \leftrightarrow 3 \mathrm{Sn}_{\mathrm{Sn}}^{\times}+4 \mathrm{P}_{\mathrm{As}}^{\times}+\mathrm{V}_{\mathrm{Sn}}^{\times} \\
& (\rightarrow 4 \text { SnAs) }
\end{aligned}
$$

Как следует из данных представленных на рис. $1 b$, сплав с содержанием 90 мол. \% SnAs является однородным, то есть растворимость фосфора в $\mathrm{SnAs}$ вдоль разреза $\mathrm{SnAs}-\mathrm{Sn}_{0.43} \mathrm{P}_{0.57}$ больше по сравнению с предыдущим разрезом. C уменьшением молярной доли моноарсенида олова до 85 мол.\% сплав становится гетерофазным, на дифрактограмме появляются рефлексы, отвечающие присутствию $\alpha$-твердого раствора.

Наибольшее отклонение от стехиометрического состава в сторону недостатка олова наблюдается при вхождении фосфора в решетку SnAs в направлении разреза $\mathrm{SnAs}-\mathrm{SnP}_{3}$ :

$$
\mathrm{SnP}_{3} \leftrightarrow \underset{(\rightarrow 3 \mathrm{SnAs})}{\mathrm{Sn}_{\mathrm{Sn}}^{\times}+3 \mathrm{P}_{\mathrm{As}}^{\times}+2 \mathrm{~V}_{\mathrm{Sn}}^{\times}}
$$

Этому направлению отвечает и наибольшая протяженность области гомогенности: сплав, содержащий 70 мол. \% SnAs, является однородным, на его дифрактограмме фиксируются линии арсенида олова, смещенные в сторону больших углов (рис. 1c).

Введение фосфора в виде индивидуального вещества должно способствовать формированию вакансий олова:

$$
\begin{aligned}
\mathrm{P} \leftrightarrow \mathrm{P}_{\mathrm{As}}^{\times}+\mathrm{V}_{\mathrm{Sn}}^{\times} & (\rightarrow \mathrm{SnAs})
\end{aligned}
$$



Рис. 2. Параметр решетки $\beta$-твердого раствора в зависимости от состава сплавов политермического сечения $\mathrm{SnAs}-\mathrm{SnP}_{3}$

[Fig. 2. Lattice parameter of a $\beta$-solid solution depending on the composition of alloys of the polythermal section

$$
\mathrm{SnAs}-\mathrm{SnP}_{3} \text { ] }
$$

Однако в этом случае, как видно из приведенного квазихимического уравнения, отклонение от стехиометрии в сторону недостатка олова уменьшается. На рис. $1 d$ представлена дифрактограмма сплава (SnAs) $)_{0.9} \mathrm{P}_{0.1}$, где наряду с линиями $\beta$-твердого раствора наблюдаются рефлексы фосфида олова $\mathrm{SnP}_{3}$, смещенные в сторону меньших углов, что свидетельствует о формировании твердого раствора на основе этой фазы $(\gamma)$.

Исследование сплавов разреза $\mathrm{SnAs}-\mathrm{SnP}_{3}$ методом рентгенофазового анализа позволило оценить межплоскостные расстояния для $\beta$-твердого раствоpa, присутствующего в сплавах, и вычислить на их основе значения параметра решетки. Концентрационная зависимость параметра решетки достаточно сложная: при концентрациях фосфида олова до 30 мол. \% параметр $a$ планомерно уменьшается, а затем изменяется крайне незначительно (рис. 2). Для начального участка характер зависимости линейный и описывается уравнением $a=-0.0012 \times+5.7222$ $(R=0.9877)$.

Согласно схеме фазовых равновесий в системe Sn-As-P, предложенной в нашей работе [11], твердый раствор на основе моноарсенида олова участвует в трех четырехфазных равновесиях, причем эвтектическое равновесие $\mathrm{L} \leftrightarrow \alpha+\beta+\gamma$ peaлизуется при температуре 818 К. По значению параметра решетки для гетерофазных сплавов (с содержанием фосфида олова 60 и 50 мол. \%, рис. 2) можно оценить, что в четырехфазном равновесии участвует твердый раствор на основе моноарсени-

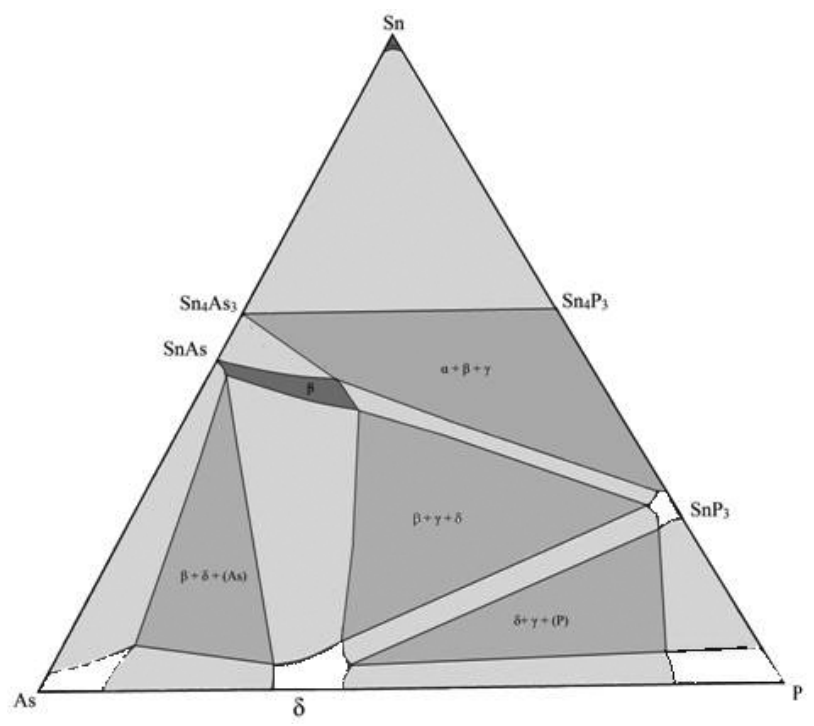

Рис 3. Схема фазового субсолидусного разграничения фазовой диаграммы системы Sn-As-P

[Fig. 3. The scheme of the phase subsolidus separation of the phase diagram of the $\mathrm{Sn}-\mathrm{As}-\mathrm{P}$ system] 
да олова, в котором около 40 \% мышьяка замещено на атомы фосфора.

На рис. 3 приведена схема фазового субсолидусного разграничения фазовой диаграммы системы Sn-As-P, на которой выделена область гомогенности твердых растворов фосфора в моноарсениде олова. Из представленной схемы видно, что область существования твердых растворов на основе моноарсенида олова имеет асимметричную форму, смещенную в сторону избытка летучих компонентов, причем максимальная растворимость фосфора отвечает направлению политермического сечения $\mathrm{SnAs}-\mathrm{SnP}_{3}$.

Результаты исследований получены на оборудовании ЦКПНО ВГУ.

\section{СПИСОК ЛИТЕРАТУРЫ}

1. Kovnir K. A., Kolen'ko Y. V., Sugata Ray, et al. // Journal of Solid State Chemistry, 2006, vol. 179, №. 7, pp. 3756-3762. DOI: https://doi.org/10.1016/j. jssc.2006.08.012

2. Kovnir K. A., Kolen'ko Y. V., Baranov A. I., et al. // Journal of Solid State Chemistry, 2009, vol. 182, № 5, pp. 630-639. DOI: https://doi.org/10.1016/j. jssc.2008.12.007
3. Shevelkov A. V. // Dalton Trans., 2010, vol. 39, p. 977. DOI: http://dx.doi.org/10.1039/B924863N

4. Семенова Г. В., Гончаров Е. Г. Твердые растворы с участием элементов пятой группьл. М.: Изд. МФТИ, $2000,160 \mathrm{c}$.

5. Семенова Г. В., Кононова Е. Ю., Сушкова Т. П. // Журн. неорган. химии, 2013, т. 58, № 9, с. 1242-1245. DOI: http://dx.doi.org/10.7868/S0044457X13090201

6. Сушкова Т. П., Семенова Г. В., Наумов А. В., Проскурина Е. Ю. // Вестник ВГУ. Серия: Химия. Биология. Фармация, 2017, № 3, с. 30-36. http://www.vestnik. vsu.ru/pdf/chembio/2017/03/2017-03-05.pdf

7. Gokcen N. A. // Bulletin of Alloy Phase Diagrams, 1990, vol. 11, № 3, pp. 271-273. DOI: https://doi. org/10.1007/BF03029298

8. Вдовина Т. 3., Медведева 3. С. // Журн. неорган. химии, 1974, т. 19, № 8, с. 2257-2260.

9. Эмсли Дж. Элементыл. Пер. с англ., М.: Мир, 1993, $256 \mathrm{c}$.

10. Картотека дифракции на порошках Международного центра дифракционных данных (ICDD PDF2), 2012.

11. Семенова Г. В., Сушкова Т. П., Тарасова Л. А., Проскурина Е. А. // Конденсированные среды и межфазные границы, 2017, т. 19, № 3, с. 408-416. DOI: https://doi.org/10.17308/kcmf.2017.19/218

\title{
SOLUBILITY OF PHOSPHORUS IN TIN MONOARSENIDE
}

\author{
(C) 2017 G. V. Semenova, T. P. Sushkova, E. N. Zinchenko, S. V. Yakunin \\ Voronezh State University, 1, Universitetskaya pl., 394018 Voronezh, Russia \\ e-mail: semen157@chem.vsu.ru
}

Received 28.08.2018

\begin{abstract}
The $\mathrm{Sn}-\mathrm{As}-\mathrm{P}$ system is characterized by the presence of unlimited solid solutions $\left(\mathrm{Sn}_{4} \mathrm{As}_{3}\right)_{x}\left(\mathrm{Sn}_{4} \mathrm{P}_{3}\right)_{1-x}(\alpha$-phase) and solid solutions based on tin monoarsenide ( $\beta$-solid solutions) and $\mathrm{SnP}_{3}$ phosphide ( $\gamma$-solid solutions). The solubility of phosphorus in SnAs depends not only on temperature, but also on the direction of the polythermal cross section, i.e. from that in the form of an individual simple substance or in the form of any of the tin phosphides, the doping component is introduced. In the present work, the configuration of the region of existence of solid solutions of phosphorus in tin monoarsenide was investigated. Alloys of $\mathrm{SnAs}-\mathrm{Sn}_{4} \mathrm{P}_{3}: \mathrm{SnAs}-\mathrm{Sn}_{0.43} \mathrm{P}_{0.57} ; \mathrm{SnAs}-\mathrm{SnP}_{3}$ and SnAs - P polythermal sections, annealed at $753 \mathrm{~K}$, were studied by X-ray phase analysis, the concentration range of the existence of a homogeneous solid solution was established, the parameters of the crystal structure were determined. The construction of quasi-chemical schemes for the doping of tin monoarsenide showed that with the introduction of $\mathrm{SnP}_{3}$, the greatest deviation from stoichiometry towards the lack of tin is observed. The highest solubility of phosphorus, reaching $30 \mathrm{~mol} \%$ corresponds to this cut. Thus, the region of existence of solid solutions based on tin monoarsenide has an asymmetric shape shifted toward an excess of volatile components, and the maximum solubility of phosphorus corresponds to the direction of the polythermal section $\mathrm{SnAs}-\mathrm{SnP}_{3}$. Taking into account the phase equilibrium scheme of the $\mathrm{Sn}-\mathrm{As}-\mathrm{P}$ ternary system and the obtained data on the configuration of the region of existence of homogeneous solid solutions of phosphorus in the tin monoarsenide, a phase subsolidus demarcation scheme of the phase diagram of this system was carried out.
\end{abstract}

Keywords: phase equilibria, solid solutions, $\mathrm{Sn}-\mathrm{As}-\mathrm{P}$ system.

DOI: https://doi.org/10.17308/kcmf.2018.20/639 


\section{ACKNOWLEDGMENTS}

The research results were obtained using the equipment of the Center for Collective Use of Equipment Voronezh State University

\section{REFERENCES}

1. Kovnir K. A., Kolen'ko Y. V., Sugata Ray, et al. Journal of Solid State Chemistry, 2006, vol. 179, no. 7, pp. 3756-3762. DOI: https://doi.org/10.1016/j. jssc.2006.08.012

2. Kovnir K. A., Kolen'ko Y. V., Baranov A. I., et al. Journal of Solid State Chemistry, 2009, vol. 182, no. 5, pp. 630-639. DOI: https://doi.org/10.1016/j.jssc.2008.12.007

3. Shevelkov A. V. Dalton Trans., 2010, vol. 39, p. 977. DOI: http://dx.doi.org/10.1039/B924863N

4. Semenova G. V., Goncharov E. G. Solid Solutions Involving Elements of the Fifth Group. Moscow, MFTI Publ., 2000, 160 p. (in Russ.)

5. Semenova G. V., Kononova E. Yu., Sushkova T. P. Russian J. of Inorganic Chemistry, 2013, vol. 58, no. 9, pp.
1112-1114. DOI: https://doi.org/10.1134/ s0036023613090192

6. Sushkova T. P, Semenova G. V., Naumov A. V., Proskurina E. Yu. Bulletin of VSU. Series: Chemistry. Biology. Pharmacy, 2017, no. 3, pp. 30-36. Available at: http://www.vestnik.vsu.ru/pdf/chembio/2017/03/2017-0305.pdf (in Russ.)

7. Gokcen N. A. Bulletin of Alloy Phase Diagrams, 1990, vol. 11, no. 3, pp. 271-273. DOI: https://doi. org/10.1007/BF03029298

8. Vdovina T. Z., Medvedeva Z. S. Russian J. of Inorganic Chemistry, 1974, vol. 19, no. 8, pp. 2257-2260. (in Russ.)

9. Emsley J. Elements. Second Edition. Oxford University Press, Oxford, 1991.

10. Powder Diffraction File for the International Diffraction Data Center (ICDD PDF2), 2012.

11. Semenova G.V., Sushkova T.P, Tarasova L.A., Proskurina E.Yu. // Condensed Matter and Interphases, 2017, vol. 19, no. 3, pp. 408-416. DOI: https://doi. org/10.17308/kcmf.2017.19/218 (in Russ.)
Семенова Галина Владимировна - д. х. н., профессор, профессор кафедры общей и неорганической химии, Воронежский государственный университет, Воронеж, Россия; тел.: +7 (473) 2208610 , e-mail: semen157@chem.vsu.ru

Сушкова Татьяна Павловна - к. х. н., доцент, доцент кафедры общей и неорганической химии, Воронежский государственный университет, Воронеж, Россия; тел.: +7 (473) 2208610, e-mail: sushtp@ yandex.ru

Зинченко Елена Николаевна - инженер ЦКПНО ВГУ, Воронежский государственный университет, Воронеж, Россия; тел.: +7(919) 1875774, e-mail: zinchenko@vsu.ru

Якунин Сергей Викторович - студент - магистрант кафедры общей и неорганической химии, Воронежский государственный университет, Воронеж, Россия; тел.: +7 (915) 5456094, e-mail: sergey. yakunin.96@mail.ru
Galina V. Semenova — Dr. Sci. (Chem.), Full Professor, Department of General and Inorganic Chemistry, Voronezh State University, Voronezh, Russia; tel.: +7(473) 2208610, e-mail: semen157@ chem.vsu.ru

Tatiana P. Sushkova - Cand. Sci. (Chem.), Assistant Professor, Department of General and Inorganic Chemistry, Voronezh State University, Voronezh, Russia; tel.: +7(473) 2208610, e-mail:sushtp@, yandex.ru

Elena N. Zinchenko - Engineer the Collective Use Center of Voronezh State University, Voronezh, Russia; tel.:+7(919) 1875774, e-mail: zinchenko@vsu.ru

Yakunin Sergei V. - Master Student, Department of General and Inorganic Chemistry, Voronezh State University; Voronezh, Russia; +7 (915) 5456094, e-mail: sergey.yakunin.96@mail.ru 